\title{
Parametric Model Order Reduction Accelerated by Subspace Recycling
}

\author{
Lihong Feng, Peter Benner, Jan G. Korvink
}

\begin{abstract}
Many model order reduction methods for parameterized systems need to construct a projection matrix $V$ which requires computing several moment matrices of the parameterized systems. For computing each moment matrix, the solution of a linear system with multiple right-hand sides is required. Furthermore, the number of linear systems increases with both the number of moment matrices used and the number of parameters in the system. Usually, a considerable number of linear systems has to be solved when the system includes more than two parameters. The standard way of solving these linear systems in case sparse direct solvers are not feasible is to use conventional iterative methods such as GMRES or CG. In this paper, a fast recycling algorithm is applied to solve the whole sequence of linear systems and is shown to be much more efficient than the standard iterative solver GMRES as well as the newly proposed recycling method MKR-GMRES from [10]. As a result, the computation of the reduced-order model can be significantly accelerated.
\end{abstract}

\section{INTRODUCTION}

At present, model order reduction has been widely applied to various engineering problems and has achieved much success in enhancing traditional simulation tools. However, many model order reduction techniques still need to be further improved. For an efficient model order reduction method, it is desired not only that the reduced model is as small as possible, but also that the process of obtaining the reduced model is as fast as possible. In this paper we consider the latter issue and try to speed up the process of some parametric model order reduction (PMOR) methods. We propose to apply a fast recycling algorithm SimGCRODR to accelerate the computation of the projection matrix $V$ for constructing the reduced model.

Many PMOR methods for model order reduction of parameterized systems have been proposed till now, most recent methods include [2], [11], [12], [13], [1] etc. Quite a few of these methods are based on matching moments of the parameterized system. For example, in [1], a modified Gram-Schmidt process is used to orthogonalize the moment matrices and to construct the projection matrix $V$. During the orthogonalization process, each moment matrix in the subspace has to be computed and orthogonalized against previous moment matrices. However, computing each moment

This work was supported by Alexander von Humboldt Foundation and by DFG grant BE 2174/7-1, Automatic, Parameter-Preserving Model Reduction for Applications in Microsystems Technology.

Lihong Feng is with Department of Microsystems Engineering (IMTEK), University of Freiburg, Germany lihong.feng@imtek.uni-freiburg.de

Peter Benner is with Faculty of Mathematics, Chemnitz University of Technology, Germany benner@mathematik.tu-chemnitz.de

Jan G. Korvink is with Department of Microsystems Engineering (IMTEK) and Freiburg Institute of Advanced Studies (FRIAS), University of Freiburg, Germany korvink@imtek. uni-freiburg. de matrix includes several vectors multiplied by the inverse of a matrix. If the dimension of the original parameterized system is very large, several linear systems of equations need to be solved to get the "inverse of matrix"-vector products. If there are several parameters in the system, many linear systems of equations have to be solved in order to match enough moments so that a reduced model with acceptable accuracy can be obtained. Furthermore, a direct solver such as LU factorization is not suitable for solving the linear systems of large dimensions, hence iterative methods are preferred.

However, it is known that the standard iterative solvers such as GMRES, CG can only solve one linear system at a time. Therefore, all the linear systems have to be solved independently, or in other words, each of them has to be solved from scratch. Instead of solving each linear system from scratch, we propose to solve them sequentially by a recycling algorithm which we call SimGCRO-DR, so that certain information of the previous linear systems can be reused (recycled) to speed up the computation of the current system. By this recycling technique, a certain number of matrixvector (MV) products can be saved for solving each system, and a large amount of MV products can be saved for solving all the linear systems. Since the major computation in both standard GMRES and the recycling algorithm SimGCRODR are MV products, the recycling algorithm SimGCRODR is much more efficient than the standard GMRES by saving massive MV products. Therefore, computation of the projection matrix $V$ is sped up by replacing GMRES with SimGCRO-DR in the modified Gram-Schmidt process in [1].

It is worth pointing out that the proposed recycling algorithm SimGCRO-DR can also be easily applied to other PMOR methods in [2], [11], [12], [13], which include solving successive linear systems when the system dimension is very large. To be clear, we use the method in [1] in this paper as an example to explore the usage of the recycling algorithm SimGCRO-DR.

Conventional block methods [3], [4] were also developed to enhance the efficiency of standard iterative solvers like GMRES or CG. Unfortunately, these methods require that the right-hand sides of all the linear systems are available simultaneously, whereas the linear systems considered here can only appear sequentially rather than simultaneously. Different algorithms [5], [8], [9], [10] have also been proposed to deal with the linear systems whose right-hand sides are not available simultaneously. However, the method in [9] is limited to solving linear systems with symmetric definite coefficient matrices, which is not applicable to the linear systems with general nonsymmetric coefficient matrices. While the method in [5] can solve linear systems 
with nonsymmetric coefficient matrices, it requires that the solutions of neighboring linear systems are closely related to each other. The solutions of the linear systems in PMOR nevertheless do not possess such a property. A recycling algorithm GCRO-DR is proposed in [8] for solving linear systems in the form of $A_{i} x=b_{i}, i=1,2, \ldots, l$. It is suitable for nonsymmetric $A_{i}$, and with no special limitation on each linear system. The current algorithm has the disadvantage of being complicated for the case we considered here. We propose a simplified version SimGCRO-DR based on GCRODR in [8] and apply it to the PMOR method in [1]. We also compare SimGCRO-DR with the newly proposed recycling method MKR-GMRES in [10]. The numerical simulation results in Section IV shows that SimGCRO-DR is more efficient than MKR-GMRES for very large-scale systems.

In the next section, besides introducing the PMOR method in [1], we describe the origin of the linear systems to be solved by recycling algorithms. In Section III, we present the recycling method GCRO-DR in [8] based on which a simplified algorithm SimGCRO-DR is derived. The method MKRGMRES in [10] is also briefly explained for comparison. SimGCRO-DR is compared numerically with both standard GMRES and MKR-GMRES in detail in Section IV.

\section{A ROBUST PARAMETRIC MODEL ORDER REDUCTION METHOD}

PMOR methods are designed for model order reduction of parameterized systems, where the parameters of the system play an important role in practical applications. The reduced models are constructed such that all the parameters can be preserved with enough accuracy. Model order reduction for parameterized systems has been researched for years and is still an active research area at present. Although there are various PMOR methods in the literature, some of these methods can be further substantially enhanced if efficient techniques are developed accordingly. The set of recycling algorithms considered in this paper is one of the efforts in this direction. In the following, we first introduce the PMOR method in [1], then we show how to further enhance the efficiency of the method by applying the recycling algorithm SimGCRO-DR proposed in the next section.

For simplicity, we use a linear parameterized system as an example, which has the following form in the frequency domain:

$$
\begin{aligned}
\left(E_{0}+s_{1} E_{1}+s_{2} E_{2}+\ldots+s_{p} E_{p}\right) x & =B u\left(s_{p}\right), \\
y & =L^{\mathrm{T}} x,
\end{aligned}
$$

where $s_{1}, s_{2}, \ldots, s_{p-1}$ are the parameters of the system, $s_{p}$ is the frequency domain variable, which corresponds to the time $t$ for the system (1) in the time domain. $x(t) \in \mathbb{R}^{n}$ is the vector of state variables, $u \in \mathbb{R}^{d}$ and $y \in \mathbb{R}^{r}$ are, respectively, the inputs and outputs of the system. To get the reduced model, a projection matrix $V$ has to be constructed by orthogonalizing the moment matrices of system (1). Defining $B_{M}=\tilde{E}^{-1} B$, $M_{i}=-\tilde{E}^{-1} E_{i}, i=1,2, \ldots, p$ and $\tilde{E}=E_{0}+s_{1}^{0} E_{1}+s_{2}^{0} E_{2}+$ $\cdots+s_{p}^{0} E_{p}, x$ in (1) can be expanded at $s_{1}, s_{2}, \ldots, s_{p}$ around the expansion points $s_{1}^{0}, s_{2}^{0}, \cdots, s_{p}^{0}$ as below,

$$
\begin{aligned}
x & =\left[I-\left(\sigma_{1} M_{1}+\ldots+\sigma_{p} M_{p}\right]^{-1} B_{M} u\left(s_{p}\right)\right. \\
& =\sum_{i=0}^{\infty}\left(\sigma_{1} M_{1}+\ldots+\sigma_{p} M_{p}\right)^{i} B_{M} u\left(s_{p}\right) .
\end{aligned}
$$

Here $\sigma_{i}=s_{i}-s_{i}^{0}, i=1,2, \ldots, p$. We call the coefficients in the above series expansion moment matrices of the parameterized system, i.e. $B_{M}, M_{1} B_{M}, \ldots, M_{p} B_{M}, M_{1}^{2} B_{M},\left(M_{1} M_{2}+\right.$ $\left.M_{2} M_{1}\right) B_{M}, \ldots,\left(M_{1} M_{p}+M_{p} M_{1}\right) B_{M}, M_{p}^{2} B_{M}, M_{1}^{3} B_{M}, \ldots$ The corresponding moments are those moment matrices multiplied by $L^{\mathrm{T}}$ from the left. Instead of explicitly computing the moment matrices as suggested in [2], a numerically robust algorithm is proposed in [14], which combines the recursions in (3) with the modified Gram-Schmidt process to implicitly compute the moment matrices.

$$
\begin{aligned}
& R_{0}=B_{M}, R_{1}=\left[M_{1} R_{0}, \ldots, M_{p} R_{0}\right], \\
& R_{2}=\left[M_{1} R_{1}, \ldots, M_{p} R_{1}\right], \\
& \vdots \\
& R_{j}=\left[M_{1} R_{j-1}, \ldots, M_{p} R_{j-1}\right] \\
& \vdots
\end{aligned}
$$

Each moment matrix is actually several vectors multiplied by $\tilde{E}^{-1}$, and if the dimension of $\tilde{E}$ is very large, it is necessary to solve linear systems like

$$
\tilde{E} x=w_{i}, i=1,2, \ldots, l
$$

to obtain $\tilde{E}^{-1} w_{i}$, where $\tilde{E}$ is generally nonsymmetric and $w_{i}$ is a vector. Moreover, if quite a few of the moment matrices need to be computed (this is normal when system (1) contains more than 2 parameters), the number $l$ of the linear systems in (4) will be very large. By looking into the above recursions, it is obvious that the right-hand sides of the linear systems cannot be available simultaneously. Systems in (4) can be simply solved one after another by standard iterative methods such as GMRES. However, it is possible to speed up the standard iterative methods by using more efficient methods. In the next section, we will review the recycling algorithm from [8] and derive a simplified algorithm SimGCRO-DR, which can solve the sequence of linear systems (4) more efficiently than GMRES.

\section{SOLVING LINEAR SYSTEMS BY A RECYCLING ALGORITHM}

A recycling algorithm GCRO-DR is proposed in [8], which is developed for sequential linear systems $A_{i} x=b_{i}, i=$ $1,2, \ldots, l$ with different $A_{i}$ and $b_{i}$. GCRO-DR combines the idea of GCRO method in [6] and that of the GMRESDR method in [7]. When GCRO-DR is used to solve a single system, it is algebraically equivalent to GMRES-DR. A recycling technique is used for different linear systems, i.e. when the current system $A_{i} x=b_{i}(i>1)$ is solved, the harmonic Ritz vectors of $A_{i-1}$ are recycled to modify the initial guess $x_{0}$ of $A_{i} x=b_{i}$. By recycling these harmonic Ritz vectors, GCRO-DR can solve the linear systems by far fewer MV products than the standard method GMRES. Therefore it is much more efficient than GMRES especially for solving 
many linear systems (see [8] for the theoretical details of the algorithm). However this algorithm is complicated for sequences of linear systems with the same coefficient matrix as in (4). We modify several steps in GCRO-DR, and present a simplified algorithm SimGCRO-DR(m,k) which is tailored for the sequence in (4). We describe SimGCRO-DR $(m, k)$ in Algorithm 1, and explain the basic idea of the algorithm in the subsequent text.

Algorithm 1: SimGCRO-DR Solving the $i$ th system in the sequence of linear systems $A x=b_{i}, i=1,2, \ldots, l$ :

1. Choose $m$, the maximum size of the subspace, and $k$ the desired number of the harmonic Ritz vectors of $A$. tol is the convergence tolerance. $x_{0}$ is the initial guess, and $r_{0}=b_{i}-A x_{0}$ is the initial error. Set $j=1$.

2. IF $C$ and $U$ are defined (from solving a previous system) then

3. $x_{1}=x_{0}+U C^{H} r_{0}$;

4. $r_{1}=r_{0}-C C^{H} r_{0}$.

5. ELSE

6. run $m$ steps of GMRES, generating a $(m+1) \times m$ matrix $\tilde{H}_{m}$, a $n \times(m+1)$ matrix $V_{m+1}$ and $x_{1}, r_{1}$.

7. Compute $k$ smallest harmonic Ritz vectors of $A$, i.e. $k$ eigenvectors $\xi_{j}$ of:

$\left(H_{m}+h_{m+1, m}^{2} H_{m}^{-H} e_{m} e_{m}^{H}\right) \xi_{j}=\lambda_{j} \xi_{j}$ associated with the $k$ smallest magnitude eigenvalues and store in $P$. Here $H_{m}$ is the $m \times m$ upper block in $\tilde{H}_{m}, h_{m+1, m}$ is the $(m+1, m)$ entry in $\tilde{H}_{m}$ and $e_{m} \in \mathbb{R}^{m}, e=[0,0, \ldots, 1]^{T}$.

8. $\tilde{Y}=V_{m} P ; V_{m}$ contains the first $m$ columns in $V_{m+1}$

9. Let $[Q, R]$ be the reduced $\mathrm{QR}$-factorization of $\tilde{H}_{m} P$;

10.

11.

12.

13.

14. run $m-k$ steps of Arnoldi algorithm with the linear operator $\left(I-C C^{H}\right) A$, letting $v_{1}=r_{j-1} /\left\|r_{j-1}\right\|_{2}$ and generating a $(m-k+1) \times(m-k)$ matrix $\tilde{H}_{m-k}$, a $n \times(m-k+1)$ matrix $V_{m-k+1}$, and $B_{m-k}=$ $C^{H} A V_{m-k}$, likewise, $V_{m-k}$ is the first $m-k$ columns in $V_{m-k+1}$.

15. Step 16-Step 20 compute $x_{j}$ whose residual $r_{j}$ is minimized over the subspace spanned by $\left[\begin{array}{ll}\tilde{U}_{k} & V_{m-k}\end{array}\right]$. Here $\tilde{U}_{k}=U D_{k}, D_{k}$ is a diagonal scaling matrix such that each column in $\tilde{U}_{k}$ has unit 2-norm.

16. Let $G_{m}=\left[\begin{array}{rr}D_{k} & B_{m-k} \\ 0 & \tilde{H}_{m-k}\end{array}\right]$.

17. $\hat{V}_{m}=\left[\tilde{U} V_{m-k}\right] ; \hat{W}_{m+1}=\left[C V_{m-k+1}\right]$;

18. Solve $\min \left\|\hat{W}_{m+1}^{H} r_{j-1}-G_{m} y\right\|_{2}$ for $y$.

19. $x_{j}=x_{j-1}+\hat{V}_{m} y$;

20. $r_{j}=r_{j-1}-\hat{W}_{m+1} G_{m} y$.
21. IF solving the first system i.e. $i=1$, then modify the $k$ harmonic Ritz vectors of $A$, i.e., compute $k$ eigenvectors $\xi_{t}$ of $G_{m}^{H} G_{m} \xi_{t}=\lambda_{t} G_{m}^{H} \hat{W}_{m+1}^{H} \hat{V}_{m} \xi_{t}$ associated with the $k$ smallest magnitude eigenvalues $\lambda_{t}$ and store in $P$.

22. $\quad \tilde{Y}=\hat{V}_{m} P$.

23. Let $[Q, R]$ be the reduced $\mathrm{QR}$-factorization of $G_{m} P$;

24. $C=\hat{W}_{m+1} Q$;

25. $U=\tilde{Y} R^{-1}$.

26. END IF

27. END WHILE.

When solving the first system, Algorithm 1 produces not only the solution $x$, but also the $k$ harmonic Ritz vectors of $A$, which are stored in $\tilde{Y}$ and will be reused for the latter systems.

Let us consider the beginning of the algorithm. Step 1 . is nothing special. Step 2. is to see whether it is the first system or latter systems. If we are solving the first system, we go directly to Step 6., where the first approximate solution $x_{1}$ is computed by running $m$ steps of GMRES and $\tilde{H}_{m}$ is generated. The $k$ harmonic Ritz vectors of $A$ are computed in Step 7. and stored in $\tilde{Y}$. The matrices $C$ and $U$ are computed in Step 9. and Step 10., where $C=A U$ and the columns in $U$ span the same subspace as the one spanned by the columns in $\tilde{Y}$. The relations between $U, \tilde{Y}$ and $C$ will be used to compute solutions $x_{j}, j>1$ in the WHILE loop. Because the operator $\left(I-C C^{H}\right) A$ is used in step. 14, the matrix $V_{m-k+1}$ computed by the Arnoldi algorithm is orthogonal to $C$. This relation guarantees that the harmonic Ritz vectors of $A$ or the columns in $\tilde{U}$ are independent of the columns in $V_{m-k+1}$, so that $\hat{V}_{m}$ in Step 17. has full column rank. Step 18. to Step 20. compute $x_{j}$ whose residual is minimized over the subspace spanned by the columns in $\hat{V}_{m}$. Therefore $r_{j}$ is actually minimized over the subspace jointly spanned by the $k$ harmonic Ritz vectors of $A$ and the $m-k$ vectors (columns in $V_{m-k}$ ) computed by Arnoldi. The harmonic Ritz vectors of $A$ are further modified in Step 21 to Step 25 till the final solution is found. From now on, the harmonic Ritz vectors of $A$ stored in $U$ will remain unchanged and will be recycled for the $i$ th $(i>1)$ linear system.

If we are solving the $i$ th system for $i>1, x_{0}$ is first modified to $x_{1}$ so that $r_{1}$ is minimized over the subspace spanned by the columns in $U$; therefore $x_{1}$ is in fact the minimized solution over the subspace spanned by the $k$ harmonic Ritz vectors of $A$. The harmonic Ritz vectors of $A$ are recycled in this way. After $x_{0}$ is modified, the algorithm is the same as solving the first system except that Step 21 to Step 25 will never be implemented.

The idea of taking the harmonic Ritz vectors into the Krylov subspace generated by Arnoldi comes from the method GMRES-DR. It is a method more efficient than GMRES especially for linear systems having extreme eigenvalues, e.g. eigenvalues of very small magnitude [7]. Therefore, in algorithm GCRO-DR and SimGCRO-DR(m,k), GMRESDR rather than GMRES is employed to compute $x_{j}$. In this 
way, SimGCRO-DR(m,k) can not only speed up GMRES (by GMRES-DR) for solving a single system, but also further accelerate GMRES-DR by recycling harmonic Ritz vectors when solving the whole sequence of linear systems.

It is clear from Algorithm 1 that the $k$ harmonic Ritz vectors of $A$ correspond to the $k$ eigenvectors of a small matrix ( $m$ is small). In addition, the harmonic Ritz vectors need only to be computed in the iterations solving the first system. Therefore the overall computational complexity of the harmonic Ritz vectors is very small and can be neglected compared with the computational complexity of solving the whole sequence of linear systems.

The main differences between GCRO-DR and SimGCRODR are:

1) In SimGCRO-DR, the harmonic Ritz vectors of $A$ are computed only in the first system, they will remain unchanged for the $i$ th system, $i>1$. In GCRO-DR, new harmonic Ritz vectors have to be computed and modified for each system.

2) In SimGCRO-DR, the modifications of the initial guess $x_{0}$ in Step 3. and Step 4. are also simplified compared with GCRO-DR, because $U$ and $C$ can be directly recycled without being recomputed. However in GCRODR, a QR factorization of a matrix with $n$ rows ( $n$ is the dimension of $A_{i}$ ) has to be performed for the $i$ th $(i>1)$ system in order to obtain the new $U$ and $C$.

3) As a result, the many corresponding $Q R$ factorizations in GCRO-DR [8] for new harmonic Ritz vectors, new $U$ and $C$ are not necessary in SimGCRO-DR. Therefore, SimGCRO-DR $(m, k)$ requires significantly less computational work than GCRO-DR.

SimGCRO-DR $(\mathrm{m}, \mathrm{k})$ is also more efficient than a newly developed recycling algorithm MKR-GMRES in [10], where the Krylov subspace generated by Arnoldi is recycled. However, the dimension of the Krylov subspace increases fast with the number of the linear systems. As a result, the algorithm becomes very slow because of the quick inflation of the Krylov subspace. MKR-GMRES for solving sequence of linear system $A x=b_{i}, i=2, \ldots, l$ is described in Algorithm 2.

Algorithm 2: MKR-GMRES solving the $i$ th system in the sequence of linear systems $A x=b_{i}, i=1,2, \ldots, l$.

1. Calculate pseudo-inverse $P^{+}$of $P^{i}$. If $i=1$, let $P^{1}=0$ and its $P^{+}=0$.

2. Let $\mathscr{P}_{s}=P^{i} P^{+}$.

3. Calculate $r=\left(I-\mathscr{P}_{s}\right) b_{i}$ and $x=V^{i} P^{+} b_{i}$.

4. Generate Krylov subspace $V_{m}$ using Arnoldi process $\left(I-\mathscr{P}_{s}\right) A V_{m}=V_{m+1} \tilde{H}_{m}$.

5. Solve least squares problem $y=\arg \min \left\|r-A\left[V^{i}, V_{m}\right] y\right\|$.

If the error is satisfactory, go to step 6, otherwise let $m=m+1$ and go to step 4 .

6. Save $V^{i+1}=\left[V^{i}, V_{m}\right], P^{i+1}=A V^{i+1}$ for the next system, and let $x=x+V^{i+1} y$ be the solution.

In Algorithm 2, besides that $P^{i}$ has $n$ rows ( $n$ is the dimension of $A$ ), the columns in $P^{i}$ also grow fast with the number of linear systems, as can be seen from Step 6 . Therefore the computation of the pseudo-inverse $P^{+}$becomes more and more difficult due to the column growth in $P^{i}$. Similarly, Step 5. also becomes difficult to be computed due to the increase of the columns in $V^{i}$. Unfortunately in order to recycle the Krylov subspace $V^{i}$, the dimensional increase in both $P^{i}$ and $V^{i}$ is inevitable. This makes the algorithm not as efficient as expected. We illustrate the above problem by numerical simulations in the next section and show that MKR-GMRES is less efficient than SimGCRO-DR(m,k).

\section{SIMULATION RESULTS}

In this section, the proposed algorithm SimGCRO$\mathrm{DR}(\mathrm{m}, \mathrm{k})$ is compared with GMRES and MKR-GMRES by applying them to a sequence of linear systems arising from PMOR of a parameterized system as below,

$$
\begin{aligned}
E d x(t) / d t & =\left(A-h_{t} A_{t}-h_{s} A_{s}-h_{b} A_{b}\right) x+B \\
y(t) & =C x(t),
\end{aligned}
$$

where $h_{t}, h_{s}, h_{b}$ are the parameters of the system. Such a system results from finite element discretization of a microthruster unit (Fig. 1). The model ${ }^{1}$ is as a generic example of a device with a single heat source when the generated heat dissipates through the device to the surroundings. The exchange between surrounding and the device is modeled by convection boundary conditions with different film coefficients at the top, $h_{t}$, bottom, $h_{b}$, and the side, $h_{s}$. The dimension of the system (5) is $n=4257$. The expression of (5) in frequency domain is

$$
\begin{aligned}
s E x(s) & =\left(A-h_{t} A_{t}-h_{s} A_{s}-h_{b} A_{b}\right) x(s)+B u(s) \\
y(s) & =C x(s) .
\end{aligned}
$$

Here $s$ is the variable in frequency domain; therefore there are totally 4 parameters in the system. Following the PMOR method introduced in Section II, the sequence of linear system are $\tilde{E} x=w_{i}, i=1,2, \ldots, l$, where $\tilde{E}=-A+s^{0} E+$ $h_{t}^{0} A_{t}+h_{s}^{0} A_{s}+h_{b}^{0} A_{b}$ and it is nonsymmetric. The number of linear systems depends on how many moment matrices of the system are used. For this example, 600 linear systems are solved to match enough moments and guarantee the accuracy of the reduced model. We take expansion points $s^{0}=1, h_{t}^{0}=$ $h_{s}^{0}=h_{b}^{0}=1$ and the reduced model is of dimension $q=325$. All the simulations are run with MATLAB 2007b.

We employ the same comparison criterion as the one used in [8] to compare $\operatorname{SimGCRO-DR(m,k)}$ with GMRES, i.e. the MV products used in the algorithm for solving each linear system. This is a reasonable criterion because the MV products only depend on the algorithm itself but not on the computer. Furthermore, the number of MV products constitutes the main computation in both algorithms, which in some sense, corresponds to the iteration steps. Therefore it is sufficient to compare the MV products used in the algorithms. In the following text, GMRES $(\infty)$ means GMRES without restarts and GMRES(m) means the one with restarts.

\footnotetext{
${ }^{1}$ For detailed description of the model, please refer to URL: http://www.imtek.uni-freiburg.de/simulation/benchmark/.
} 


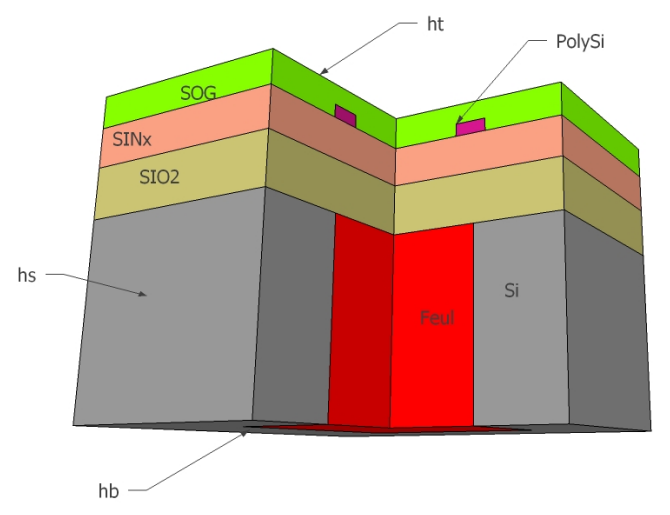

Fig. 1. A model of the microthruster unit

We compare SimGCRO-DR(m,k) with both GMRES $(\infty)$ and GMRES(m).

As is analyzed in Section III, the main computational complexity in MKR-GMRES is constituted by the computation of the pseudo-inverse of a large matrix and the least squares solution of a large dimensional problem, therefore it is not reasonable to compare MKR-GMRES with SimGCRO$\mathrm{DR}(\mathrm{m}, \mathrm{k})$ with MV products. We compare the two methods by CPU time spent on solving each system. Although the CPU time varies from computer to computer, the simulation results are clear enough to differ the two methods because of the big difference of CPU time between the two methods when they are run on the same computer.

We use the same convergence criteria $\left(t o l=10^{-7}\right)$ and preconditioning for all the above three methods. In the following simulation results, we apply two different incomplete LU factorizations as the preconditioners: in MATLAB notation, one is $\operatorname{luinc}(\cdot, 0.005)$, the other is $\operatorname{luinc}(\cdot, 0.001)$. Once the preconditioners are applied, the number of MV products will be counted based on the preconditioned algorithm. Because the two factors $L$ and $U$ are triangular matrices, it is reasonable to count one time of preconditioning by $L$ and $U$ as two MV products for simplicity.

In comparing SimGCRO-DR and restarted GMRES, we let the solution produced by both methods be minimized over the subspace of the same dimension, i.e. we use the same $m=40$ in SimGCRO-DR(m,k) and GMRES(m). We first compare preconditioned SimGCRO-DR(m,k) with preconditioned GMRES $(\infty)$ in Fig. 2. The results of GMRES(m) are presented separately in Fig. 3.

Fig. 2 shows that the number of MV products used in SimGCRO-DR(40,30) for solving most of the linear systems is less than half of those used in $\operatorname{GMRES}(\infty)$. Comparing Fig. 2 with GMRES(40) in Fig. 3, we see that for each linear system, GMRES(40) uses 10x more MV products than GMRES $(\infty)$, and GMRES(40) uses at least 20x more MV produtcs than SimGCRO-DR(40,30). We also present the results of GMRES(80) which uses much less MV products than GMRES(40), however it is still much less efficient than SimGCRO-DR(40,30). Totally 600 linear systems are

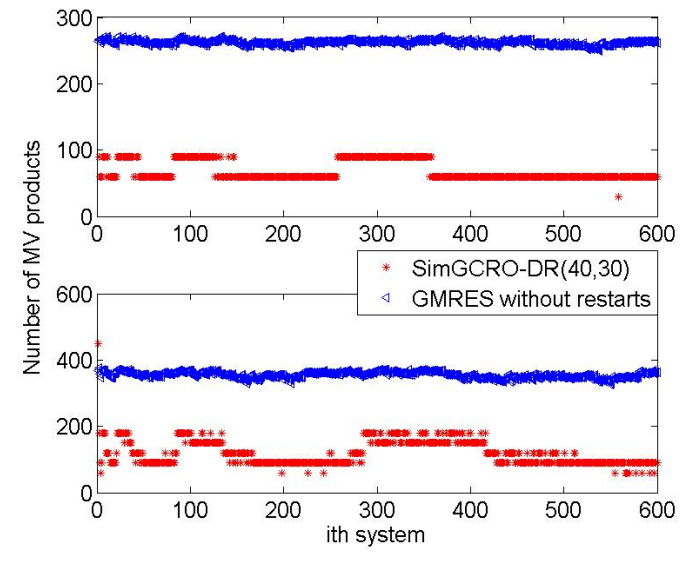

Fig. 2. SimGCRO-DR vs. GMRES( $\infty)$, top: $\operatorname{luinc}(\cdot, .001)$, bottom: $\operatorname{luinc}(\cdot, .005)$.

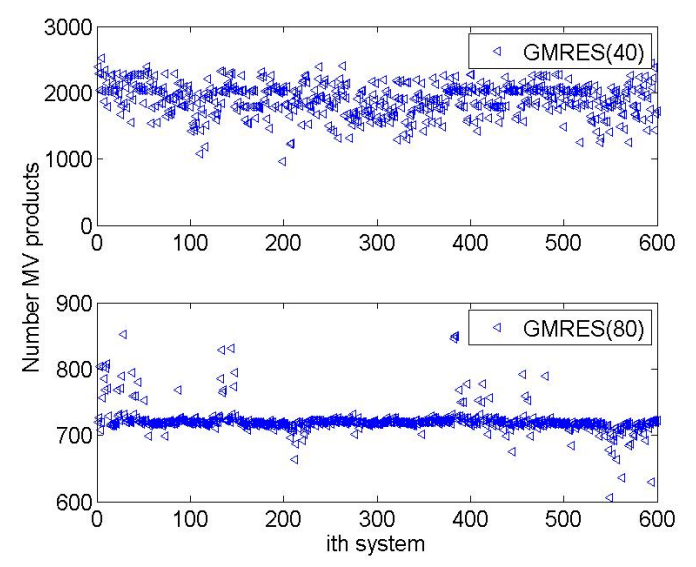

Fig. 3. GMRES(m), luinc $(\cdots, .001)$

solved to get the reduced model, therefore there is a large amount of computation saved by SimGCRO-DR. The results of GMRES(m) with preconditioner $\operatorname{luinc}(\cdot, 0.005)$ are even worse, and are therefore not given here.

Fig. 4 includes the MV products used in MKR-GMRES with the preconditioner $\operatorname{luinc}(\cdot, .001)$ (the figure on the top). The number of MV products used for the first system is 2337, which is much more than other systems and so is not included in the figure for clarity. Although the number of MV products for each system in MKR-GMRES is relatively small, the corresponding CPU time becomes longer and longer, which is described by the figure on the bottom. It is not surprising from the above analysis that the main computational complexity of MKR-GMRES is not the MV products but the high dimensional pseudo-inverse computation in step 1. and the least squares solution in step 5.. The CPU time of MKR-GMRES for solving each system increases very fast with the increase of the linear systems, whereas the CPU time used in SimGCRO-DR is much less (around $1 s$ ) and remains steady.

In order to show that the use of SimGCRO-DR in the context of PMOR does not cause accuracy problems, we 

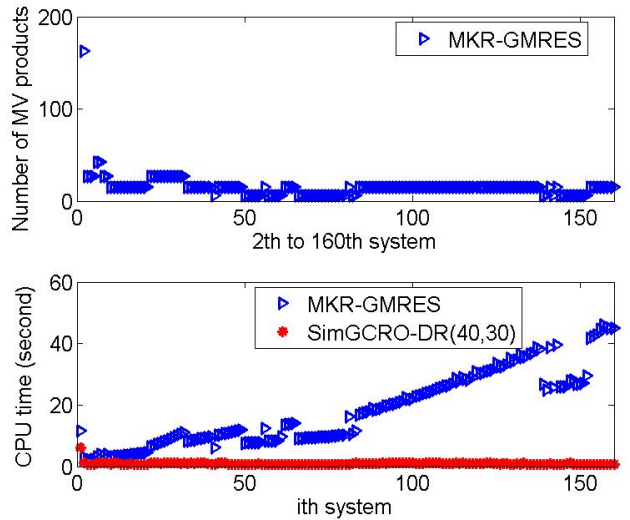

Fig. 4. Behavior of MKR-GMRES

depict the model reduction error in Fig. 5 as the 2-norm relative error between the output of the reduced model and that of the original system on the whole range of the time interval. The error is derived by combining the PMOR method in Section II with the recycling algorithm SimGCRODR. By fixing $h_{t}$ (here $h_{t}=100$ ), Fig. 5 indicates the error varying with the two parameters $h_{s}, h_{b}$. We take 529 groups of $h_{s}$ and $h_{b}$ which cover the whole range $\left[1,10^{9}\right]$. The maximum error of the reduced model with all the groups of parameters is around $10^{-7}$, and it is much smaller than the requirement of the maximum error 0.05 for real applications.

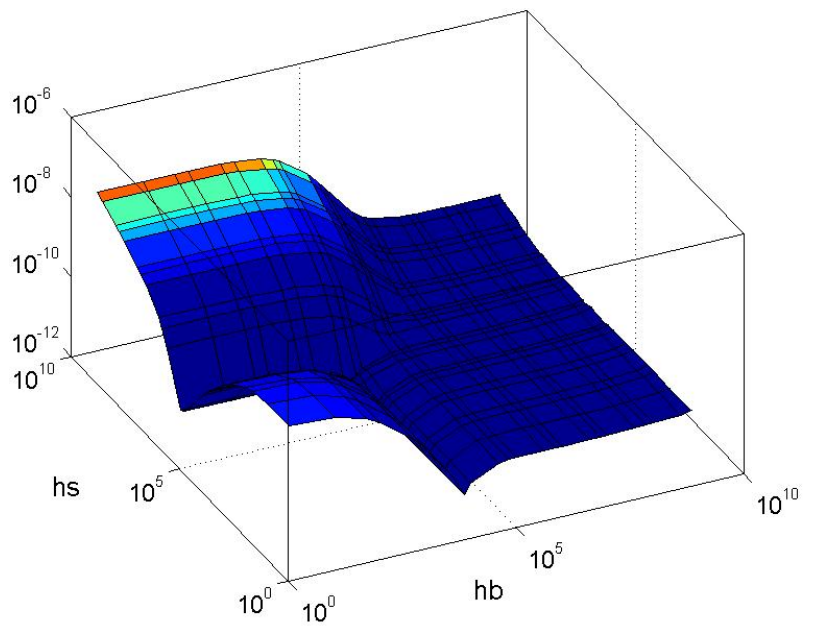

Fig. 5. Error of the reduced model by applying SimGCRO-DR

\section{CONCLUSIONS AND FUTURE WORKS}

The fast recycling algorithm SimGCRO-DR for solving successive linear systems has been applied to parametric model order reduction in the current paper. The algorithm is used to accelerate the process of PMOR, especially the part of obtaining the projection matrix $V$. It is shown that SimGCRO-DR not only outperforms the standard solver
GMRES by saving a large amount of MV products, but also is more efficient than a newly proposed recycling method MKR-GMRES [10]. The recycling algorithm can be easily integrated into the process of many other MOR techniques, where computation of a sequence of linear systems is unavoidable for large dimensional systems. Future work will focus on the following two aspects. Firstly, the recycling algorithm SimGCRO-DR will be applied to other possible fields. Secondly, a more robust recycling algorithm for solving linear systems with different coefficient matrices needs to be studied. Such linear systems arise, e.g. from multi-point expansion of the transfer function in MOR, PMOR, or from noise analysis of RF circuits [15] etc.. It is expected that the computational complexity of frequent $Q R$ factorizations in GCRO-DR can be largely reduced.

\section{REFERENCES}

[1] L. Feng and P. Benner, "A Robust Algorithm for Parametric Model Order Reduction", In Proc. Applied Mathematics and Mechanics (ICIAM 2007), 7(1): 1021501-1021502, 2007.

[2] L. Daniel, O. C. Siong, L. S. Chay, K. H. Lee, and J. White. "A multiparameter moment-matching model-reduction approach for generating geometrically parameterized interconnect performance models". IEEE Trans. Comput.-Aided Des. Integr. Circuits Syst., 22(5): 678-693, 2004.

[3] M. Kilmer, E. Miller, and C. Rappaport, "QMR-Based projection techniques for the solution of non-Hermitian systems with multiple right-hand sides". SIAM J. Sci. Comput., 23 (3): 761-780, 2001.

[4] Y. Saad, M. Yeung, J. Erhel, and F. Guyomarc'h, "A deflated version of the conjugate gradient algorithm", SIAM J. Sci. Comput., 21 (5): 1909-1926, 2000.

[5] P. F. Fischer, "Projection techniques for iterative solution of $A \underline{x}=$ $\underline{b}$ with successive right-hand sides". Comput. Methods Appl. Mech. Engi., 163: 193-204, 1998.

[6] E. de Sturler, "Nested Krylov methods based on GCR", J. Comput. Appl. Math., 67: 15-41, 1996.

[7] R. B. Morgan, "GMRES with deflated restarting", SIAM J. Sci. Comput., 24: 20-37, 2002.

[8] M. L. Parks, E. de Sturler, G. Mackey, D. D. Johnson, and S. Maiti, "Recycling Krylov subspaces for sequences of linear systems". SIAM J. Sci. Comput., 28 (5): 1651-1674, 2006.

[9] M. Clemens, M. Helias, T. Steinmets, and G. Wimmer, "Multiple right-hand side techniques for the numerical simulation of quasistatic electric and magnetic fields". J. Comput. Appl. Math., 215: 328-338, 2008.

[10] Z. Ye, Z. Zhu, and J. R. Phillips, "Generalized Krylov Recycling Methods for Solution of Multiple Related Linear Equation Systems in Electromagnetic Analysis". In Proc. Design Automation Conference (DAC08), 682-687, 2008

[11] L. Feng, E. B. Rudnyi, and J. G. Korvink, "Preserving the film coefficient as a parameter in the compact thermal model for fast electro-thermal simulation". IEEE Trans. Comput.-Aided Des. Integr. Circuits Syst., 24(12):1838-1847, 2005.

[12] X. Li, P. Li, and L. T. Pileggi. "Parameterized interconnect order reduction with explicit-and-implicit multi-parameter moment matching for inter/intra-die variations". In Proc. International Conference on Computer-Aided Design, 806-812, 2005.

[13] Y. Li, Z. Bai, Y. Su, and X. Zeng. "Model order reduction of parameterized interconnect networks via a two-directional Arnoldi process". In Proc. International Conference on Computer-Aided Design, 868-873, 2007.

[14] L. Feng and P. Benner, "A robust algorithm for parametric model order reduction based on implicit moment matching", submitted.

[15] R. Telichevesky, K. Kundert, and J. White, "Efficient AC and noise analysis of two-tone RF circuits", In Proc. Design Automation Conference, 292-297, 1996. 